\title{
A NEW SET OF MAGNETIC SEPTA IN THE CERN PS COMPLEX
}

\author{
J. Borburgh, M. Hourican, M. Thivent, PS Division, CERN, 1211 Geneva 23
}

\section{Abstract}

Over the last few years the CERN PS Complex has been upgraded to fulfill the requirements of two major projects : (i) - producing and transferring lead ions to the SPS for high energy physics experiments and (ii) raising the PSB to PS transfer energy from 1 to $1.4 \mathrm{GeV}$ to generate the high brightness beam for the LHC. To cope with the tight demands imposed by these upgrades, ten magnetic septa, operating under vacuum in the PSB and the PS have been redesigned and progressively replaced. The new devices are bakeable, better suited to high vacuum operation and with higher performance. This paper reports the main characteristics and technological advantages of these magnets together with their present performance.

\section{INTRODUCTION}

Up to 1994 the yokes of the septa ejection magnets in the Proton Synchrotron ring were built of packets of laminations glued together with 'Araldite'. For reasons of life-time, lead ions require a better vacuum than could be achieved using the existing organic materials under vacuum. In the Booster, DC septa magnets, that were originally designed for $800 \mathrm{MeV}$ beams, have been used for $1 \mathrm{GeV}$ beams, since 1986, without modification. Because of the beam emittance requirements of the future LHC accelerator, the booster energy needs to be increased to $1.4 \mathrm{GeV}$ as from 1999. The thermal limit of the DC magnets implied redesigning all Booster ejection and transfer septa. For reconstruction of these septa magnets, a single turn pulsed magnet approach has been adopted, which requires less energy hence less cooling power and therefore is less prone to erosion in the cooling circuits. To attain the required vacuum, the laminated yoke of these pulsed magnets must first be baked at 200 ${ }^{\circ} \mathrm{C}$, despite the fact that these magnets are installed in non-bakeable accelerators. The connecting flanges of the magnet tanks should not exceed $50{ }^{\circ} \mathrm{C}$ when the magnets are being baked.

\section{CONSTRUCTION DETAILS}

To reach the objectives stated in the introduction, the entire conception of the magnets has been revised. In the following paragraphs, the most outstanding details will be described per component.

\subsection{Vacuum Tanks}

To reduce the risk of a leak developing during a bake out, cylindrical tanks have been fitted with circular UHV flanges for diameters $<200 \mathrm{~mm}$ and 'Wheeler' flanges with copper seals for the bigger diameters $(>375 \mathrm{~mm})$. The connecting flanges of the tanks to the accelerator vacuum chamber are of a conical 'quick disconnect' type with aluminium seals, as standard in the CERN PS accelerators.

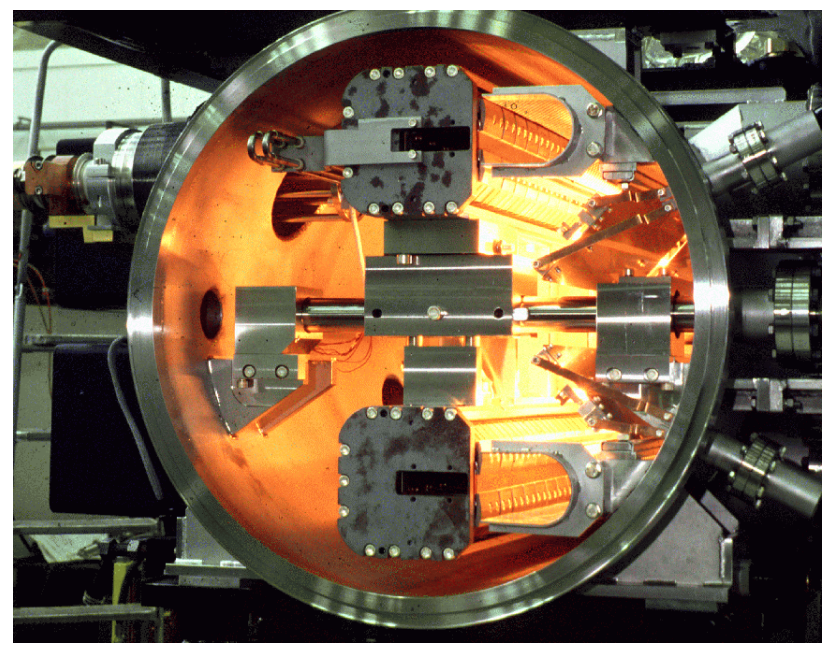

Figure 1: Vacuum Tank containing 2 ejection magnets

\subsection{Magnet yoke}

The new septa magnets, being of a pulsed type, are constructed with laminated yokes. Standard $0.35 \mathrm{~mm}$ thickness steel laminations with a $3 \%$ silicon content have been used, which are insulated on both sides with a 'Carlite' inorganic insulating coating. This solution provides a good inter-laminar resistance, and is still bakeable up to $200{ }^{\circ} \mathrm{C}$. The laminated yoke is held together in a stainless steel support by ceramic coated endplates, while sitting on ceramic bars, to ensure the electrical insulation between the yoke and the support.

\subsection{Coil}

The single-turn coils have been made of 'OFHC' copper. The cooling circuit comprises two thin-walled stainless steel tubes embedded (and brazed) in pre-machined slots in the septum conductor. This reduces erosion of the cooling circuit due to the high water speeds of up to 10 $\mathrm{m} / \mathrm{s}$. To increase the mechanical strength of the $60 \mathrm{~mm}$ gap septa conductors, a $0.5 \mathrm{~mm}$ thick stainless steel plate 
is brazed onto the outside of the septum conductor. To reduce the fringe field of the magnets, insulation has been eliminated between magnet yoke and septum conductor, while the return conductor of the coil is insulated with several layers of $0.06 \mathrm{~mm}$ and $0.12 \mathrm{~mm}$ thermally preformed 'Kapton' sheet, (see fig. 2)

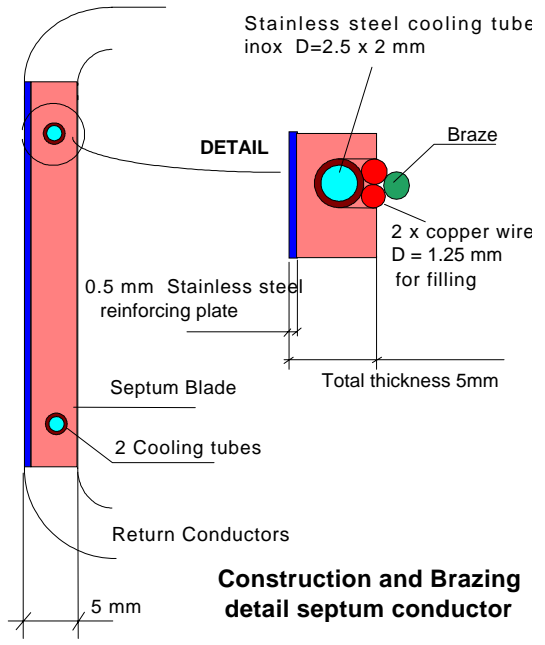

Figure 2: Cross section of septum conductor

\subsection{Coil Springs}

The complete magnet coil is held in the gap by clamping plates located outside of the magnet yoke. These insulated clamps retain the septum conductor in the gap, while a spring between the septum and rear conductor applies a mechanical force on the return conductor equal to the electromechanical force at the peak current.

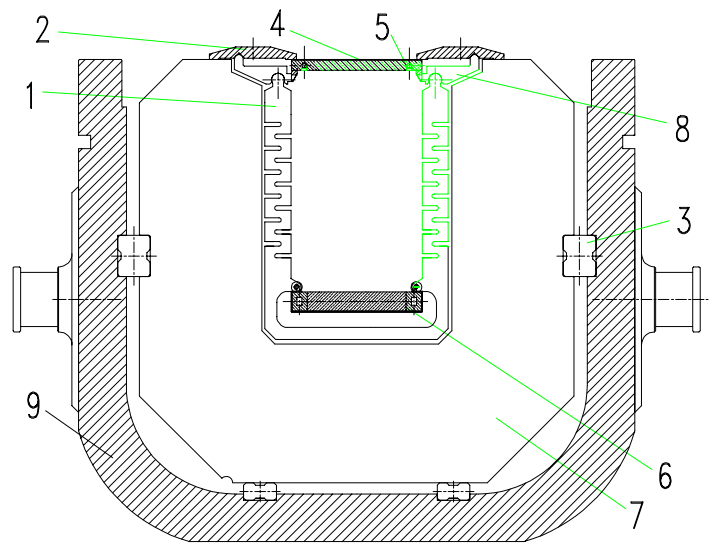

Figure 3: Cross section of magnet yoke assembly

[ 1. Damping Spring 2. Clamping Plate 3. Ceramic Support 4. Septum Conductor 5. Cooling Tube 6. Rear Conductor 7. Lamination 8. Lever 9. Stainless Steel Support ]

The springs, spaced every $45 \mathrm{~mm}$, are made of beryllium copper $(5 \% \mathrm{Be})$ suitably annealed to obtain the necessary modulus of elasticity, (see fig. 3)

\subsection{Power Feedthrough}

A water-cooled coaxial feedthrough, developed for use at $10 \mathrm{kA}_{\text {rms }}$, is used for all new pulsed septa magnets. The coaxial configuration provides the best possible symmetry in mechanical forces, (see fig. 4)

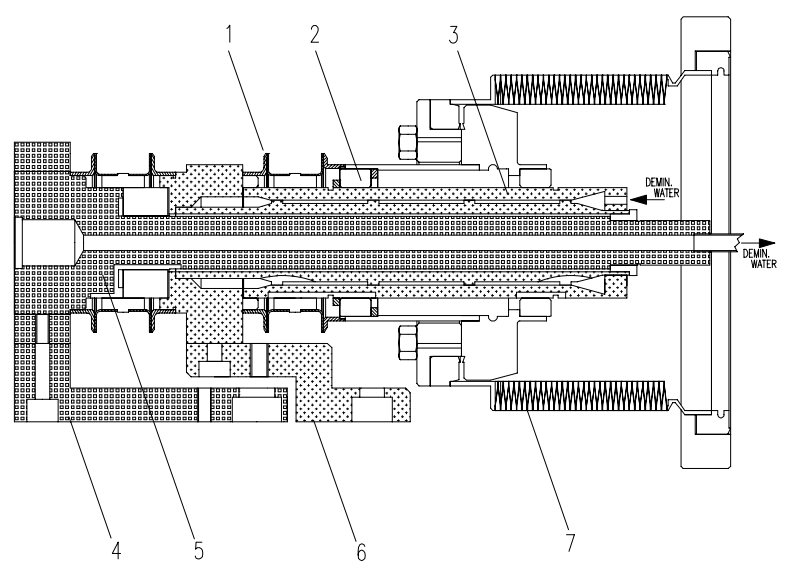

Figure 4: Cross section of power feedthrough

[ 1. Dilver / Stainless steel weld 2. Ceramic support 3. External conductor 4. Main connection 5. Central conductor 6. Main connection 7. Vacuum bellows ]

\subsection{Displacement system}

All septa magnets can be moved remotely in the radial (or vertical, in the case of the septa in the transfer line) and angular directions, while their vacuum tanks remain fixed. A variable potentiometer, linked to the mechanical displacement system, provides a measurement of the position of the magnet inside the tank. The resolution of the radial and angular systems are $0.1 \mathrm{~mm}$ and $0.1 \mathrm{mrad}$, respectively. For the initial installation, the magnet tank is aligned in all directions. In the event of a failure, the entire vacuum tank with septum magnet can be replaced without the need for realignment.

\subsection{Beam Screen}

All septa magnet tanks (except transfer lines) are equipped with RF beam screens for the orbiting beam. These screens insure the continuity of the RF impedance of the vacuum tanks, to avoid unwanted harmonics during acceleration. The beam screens are made of perforated stainless steel sheet to improve vacuum pumping speed, and are linked with the connecting flanges of the tank by means of RF contacts.

\subsection{Vacuum Equipment}

The vacuum tanks are kept under vacuum with a dedicated set of ion pumps, additional pumping being provided by titanium sublimators for the tanks installed in the accelerator rings. In view of the large surface of the magnet laminations under vacuum, and the pressure level to be obtained, it is necessary to bake out the magnets 
before commissioning. Standard off-the-shelf infra red lamps are used inside the vacuum tank, with reflectors of electro-polished stainless steel. By connecting two in series, the power consumption is reduced by a factor four, while their expected life time is dramatically increased. The temperature of the magnet is measured with ordinary $\mathrm{Cu} / \mathrm{Cn}$ (Cn-Constantan) thermocouples, and the control and regulation utilises standard temperature regulators.

\subsection{Calculations}

For the first rough estimation of the septum magnet parameters, a program was used running under Microsoft Excel developed in the CERN/PS/SEPTA section. To finalise the cross sectional design of the magnet a finite element based program called 'FLUX2D' from Cedrat/Magsoft was used. The longitudinal design was determined using the finite element based program 'OPERA3D/TOSCA' from Vector Fields, using a model developed in house to simplify the magnet model without great loss of precision. For mechanical and thermal calculations the finite element program ANSYS from Swanson Analysis Systems was used, while the cooling requirements were checked with the CERN developed program TUBE, running under Microsoft Excel.

\section{TECHNICAL SPECIFICATIONS}

The technical specifications of all septa magnets are shown in Table 1.

\section{RESULTS}

Measurements of magnetic length of the septa confirm theoretical predictions to within $0.5 \%$ of the model. The fringe fields measured are less than 1/1000 of the gap field at a $50 \mathrm{~mm}$ distance from the septum conductor, or better in case of the BESMH, (PSB Ejection) where a very low fringe field was required. After a bake out cycle, consisting of a quasi linear temperature increase of 200 ${ }^{\circ} \mathrm{C}$ over 12 hours, a 24 hour period at $200{ }^{\circ} \mathrm{C}$, and an exponential temperature decrease of approximately 48 hours, a vacuum of $6 \times 10^{-10}$ to $4 \times 10^{-9}$ mbar is achieved. Since the bake out lamps are installed with reflectors, the connecting flanges of the vacuum tanks never exceed 50 ${ }^{\circ} \mathrm{C}$, allowing the use of aluminium seals for connecting to the accelerator vacuum chambers.

\section{CONCLUSIONS}

The new set of septa magnets constructed as described in this article shows that it is possible to achieve a vacuum in the low $10^{-9}$ mbar range, even with laminated yokes under vacuum. In order to reach the low pressures specified, it is imperative to perform a pre-commissioning bakeout at $200{ }^{\circ} \mathrm{C}$. The bakeout is performed using commercially available infra red lamps using less than nominal power avoiding excessive temperatures on the vacuum chamber connecting flanges.

The septa magnets for Booster ejection, beam transfer, and PS injection allow for $1.4 \mathrm{GeV}$ operation without reaching thermal limits, since they are constructed as pulsed laminated magnets.

Table 1: Technical specifications of the septa magnets

\begin{tabular}{|l|c|c|c|c|c|c|}
\hline & BESMH & BTSMV10 & BTSMV20 & PISMH42 & PESMH16 & PESMH58 \\
\hline$L_{\mathrm{eq}}(\mathrm{m})$ & 0.95 & 1.00 & 1.00 & 0.57 & 2.18 & 0.81 \\
\hline$B_{0}(\mathrm{~T})$ & 0.354 & 0.569 & 0.525 & 0.689 & 1.20 & 1.06 \\
\hline$\delta_{B . d l}(\mathrm{~T} . \mathrm{m})$ & 0.336 & 0.566 & 0.523 & 0.390 & 2.60 & 0.860 \\
\hline $\mathrm{I}(\mathrm{kA})$ single turn coil & 7.0 & 27.3 & 25.2 & 33.1 & 28.5 & 21.1 \\
\hline E $(\mathrm{GeV})$ protons & 1.4 & 1.4 & 1.4 & 1.4 & 25.1 & 12 \\
\hline Deflection Angle (mrad) & 47 & 79 & 73 & 55 & 30 & 20 \\
\hline Gap height (mm) & 25 & 60 & 60 & 60 & 30 & 25 \\
\hline Gap width (mm) & 89 & 116 & 116 & 116 & 65 & 65 \\
\hline Septum Thickness $(\mathrm{mm})$ & 3.8 & 5 & 5 & 5 & 3 & 3 \\
\hline Rear conductor thick $(\mathrm{mm})$ & 7.6 & 8.8 & 8.8 & 8.8 & 6.0 & 6.0 \\
\hline $1 / 2$ sine pulse width $(\mathrm{ms})$ & 3.1 & 3.1 & 3.1 & 3.2 & 3.5 & 2.8 \\
\hline Water flow (1/min.) & 1.9 & 3.5 & 3.5 & 4.25 & 1.2 & 1.45 \\
\hline Year of installation & 1998 & 1999 & 1997 & 1996 & 1994 & 1994 \\
\hline
\end{tabular}

\section{REFERENCES}

[1] J.Borburgh, "2 Dimensional Finite Element Calculations On Septum Magnets with "FLUX2D" and "Opera2D"', CERN Note PS/CA/Note 97-25

[2]J.Borburgh, "3 Dimensional Finite Element Calculations On Septum Magnets with "Opera3D"”, CERN Note PS/CA/Note 97-27

[3]M.Hourican, "Remote Positioning System BTSMV20”, CERN Note PS/CA/Note 97-02

[4]J.Borburgh and M. Thivent, "SMH16-Flexible Stripline Failure Analysis with Ansy's", CERN Note PS/CA/Note 95-10 